\title{
MAINTENANCE OF MALE STERILITY IN PLANT POPULATIONS
}

\author{
M. D. ROSS* $†$ and R. F. SHAW $\ddagger$ \\ Department of Biology * and Departments of Paediatrics and Preventive Medicine $\ddagger$, Dalhousie \\ University, Halifax, Nova Scotia
}

Received 19.i.70

\section{INTRODUCTION}

THE occurrence of female plants (often called male steriles) together with hermaphrodites constitutes an interesting outbreeding mechanism known as gynodioecy (Mather, 1940). Their outbreeding results from the enforced crossing of the male steriles to the harmaphrodites. Since the male steriles are only able to produce offspring via the ovules, and not via the pollen, they are at a disadvantage compared to the hermaphrodites. It is therefore of interest to consider how male steriles may be maintained in gynodioecious populations.

Lewis (1941) has shown that the selection pressures required to maintain male sterility differ according to the mode of inheritance of the male sterility. Under monogenic inheritance, regardless of whether the allele for male sterility was recessive or dominant, male steriles were lost from a population unless they were at least twice as seed-fertile as the hermaphrodites. By contrast, when male sterility was inherited through a maternally-transmitted cytoplasm, only a slight differential seed fertility was required to maintain the male steriles.

Not much is known of the inheritance of gynodioecy. At the time of Lewis' paper gynodioecy appeared to be inherited either through the cytoplasm, or in complex ways that were not understood. Since that time cases of genic inheritance of gynodioecy have been discovered. In the grass Cortaderia richardii male sterility is inherited as a monogenic recessive (Connor, 1965), while in Origanum vulgare and in Plantago lanceolata different types of digenic inheritance of male sterility are found (Lewis and Crowe, 1956; Ross, 1969). In their discussion of the Origanum situation, Lewis and Crowe (loc. cit.) stated that under this type of digenic inheritance the male steriles were at a greater disadvantage than under monogenic inheritance.

The purpose of the present paper is to compare the selection pressures needed to maintain male sterility under monogenic inheritance with those required to maintain male sterility under digenic inheritance, with three different modes of gene interaction. In addition we are concerned with the equilibrium frequency of male steriles as a function of their relative fertility. Lewis (loc. cit.) has derived an expression governing such equilibria under monogenic inheritance of male sterility, and has found that with increasing values for the relative fertility of the male steriles, their equilibrium proportions approach a limiting value of $0 \cdot 5$. Our second problem is, then, to extend this study to the equilibria produced with digenically inherited male sterility.

* Present address: Grassland Research Institute, D.S.I.R., Palmerston North, New Zealand. 


\section{METHOD}

The method is a discrete-generation model used by Shaw (1957) to investigate the behaviour of genes affecting the sex ratio in dioecious organisms. In its present form it is inadequate for investigating problems that involve assortative mating and inbreeding. Apart from that limitation it provides a single simple method for the solution of quite diverse problems. After the procedure was published in 1957, a matrix algebra form of it was found. This will be published elsewhere. Here the method is given without proof and in its simplext, most intuitive form.

Consider two autosomal alleles $A$ and $a$ in a dioecious organism. Assume random mating, and no overlap of generations. If the frequency of genotype $A A$ is 0.25 in each sex, the frequency of the cross $A A \times A A$ is $0.25^{2}$. This value is entered into the first cell of an array called $\mathrm{Z}$, which represents the frequencies of each type of mating in the population. Similarly, frequencies of other matings such as $A A \times A a, A a \times A a$, etc., are entered into other cells in the $\mathrm{Z}$ array. If the fitness of the genotype $A A$ is 1 in each sex, the product of these fitnesses, also 1 is entered into the proper cell of a $\mathrm{W}$ or mating-type fitness array. Thus the products of the fitnesses of the genotypes represent the relative numbers of offspring left by given crosses (in the above example, the cross $A A \times A A$ ). Similarly, segregation or $\mathrm{S}$ arrays specify the segregations expected for each sex according to the mode of inheritance under study, and T arrays, whose elements are the total of the elements of the $\mathrm{S}$ arrays, are also used in the calculations. Corresponding elements in the $\mathrm{Z}, \mathrm{W}$ and $\mathrm{S}$ arrays are multiplied and summed (result 1). Similarly, corresponding elements in the $\mathrm{Z}, \mathrm{W}$ and $\mathrm{T}$ arrays are multiplied and summed (result 2). Result 1 divided by result 2 gives the frequency of the desired genotype in the next generation. The method is thus an algorithm for finding the next-generation frequencies in a population with nonoverlapping generations. This last specification is probably not a restriction of great importance, particularly in the present application.

Although originally designed for dioecious organisms, the algorithm can also be used for hermaphroditic ones. It is only necessary to set up arrays in which all genotypes appear on both axes. This is to say that the two axes do not correspond to male and female, but that genotypes $A A$ hermaphrodite, $A a$ hermaphrodite, and $a a$ hermaphrodite, appear on both. If we again consider two autosomal alleles $A$ and $a$ in a dioecious organism, where the fitness of the three genotypes $A A, A a$ and $a a$ are (for example) 1, 1, 0.5 in each sex, then the $\mathrm{W}$ array obtained by multiplying these genotypic fitnesses is

$$
\begin{aligned}
& \begin{array}{ccc}
A A & A a & a a \\
1 & 1 & 0.5
\end{array} \\
& \mathrm{~W}=\begin{array}{c}
A A \\
1 \\
1 \\
a a \\
0.5
\end{array}\left[\begin{array}{lll}
1 & 1 & 0.5 \\
1 & 1 & 0.5 \\
0.5 & 0.5 & 0.25
\end{array}\right]
\end{aligned}
$$


The elements of $\mathrm{W}$ have been obtained by multiplying the marginals, but it can also be seen by examining the elements directly that they are a measure of reproductivity of each cross. If genotype $a a$ is only half-fertile or halfviable, the fitnesses of the mating types will be as shown.

For use with gynodioecious plants the $W$ or fitness array has to be modified.

Let us consider a system in which genotype $a a$ is male sterile and genotypes $A A$ and $A a$ are hermaphrodite. Since the individuals are not divided into males and females, all genotypes appear on both axes. The $\mathrm{W}$ array can be given as

$$
\mathrm{W}=A a\left[\begin{array}{ccc}
A A & A a & a a \\
1 & 1 & 0.5 \\
1 & 1 & 0.5 \\
0.5 & 0.5 & 0
\end{array}\right]
$$

This is a mating-type approach to the problem. For organisms with monogamy it is clear and appropriate, but it may seem quite wrong for plants. What it means is this. Suppose that pairs of plants are isolated and monogamy enforced. Then cross $a a \times A$ - would be half as fertile as cross $A-\times A-$ (if we assume selfing to be absent or negligible), and cross $a a \times a a$ will be completely infertile. The scheme may still seem artificial, but it appears to give a reasonable result.

There is another approach to setting up the same $W$ array as follows.

As before, put all genotypes on both axes, but for purposes of obtaining W label the axes "fitness as males" and "fitness as females" and assign marginal values on this basis. Thus the "fitness as male" of the malesterile aa genotype is zero, although its fitness as female is as usual, one. We thus have the following array:

$$
\begin{aligned}
& \begin{array}{cccc}
\text { as females } & A A & A a & a a \\
& 1 & 1 & 1
\end{array} \\
& \text { male : } \\
& \mathrm{W}=A a \begin{array}{ll}
A A & 1 \\
a a & 0
\end{array}\left[\begin{array}{lll}
1 & 1 & 1 \\
1 & 1 & 1 \\
0 & 0 & 0
\end{array}\right]
\end{aligned}
$$

Elements of $\mathrm{W}$ are given as products of marginals as shown. The two W's look different, but they are not. They give identical results on calculation and can be shown to be algebraically equivalent in the algorithm. Subsequent discussion will, therefore, be phrased in terms of the second method.

Abbreviations used in this paper are MS for male sterile and $\mathrm{H}$ for hermaphrodite.

\section{Results}

Preliminary studies were made with monogenically inherited male sterility, under the assumptions of random mating and non-overlap of generations, as given above. Our results were similar to those of Lewis (1941). 
With recessive male sterility where the relative fertility $(w)$ of the male steriles was one, all of a variety of starting values for the proportions of male steriles (ranging from 0.9801 to 0.0001 ) declined by generation 500 to values so low

TABLE 1

Initial proportions

of Initial gene

male frequencies

steriles

$0 \cdot 6561$

$0 \cdot 2401$

$0 \cdot 0625$

$p m s_{1}, p m s_{2}$

$w=1 \quad w=2 \quad w=2 \cdot 01$

$w=3$

$w=10$

$w=100$

$\begin{array}{ll}0 \cdot 9,0 \cdot 9 & 0 \cdot 0 \\ 0 \cdot 7,0 \cdot 7 & 0 \cdot 0\end{array}$

$0.000197 * 0.012860 * 0.014874 *$

$0 \cdot 25 * * *$

$0.444444 * * * \quad 0.494949 * * *$

$0.5,0.5$

$0.000195 * 0.012747 * 0.014762 *$

$0 \cdot 25 * * *$

$0.25 * * *$

$0.444444 * * * 0 \cdot 494949 * * *$

$0 \cdot 3,0 \cdot 3$

$0.000191 * 0.012100 * 0.014097 *$

$0 \cdot 25 * * *$

$0 \cdot 444444 * * * \quad 0.494949 * * *$

$0 \cdot 0001$

$0 \cdot 1,0 \cdot 1$

0.000050* 0.000099* 0.000100089**

$0 \cdot 000455 * *$

$0 \cdot 444444 * * * \quad 0.494949 * * *$

* Decreasing. ** Increasing. *** At equilibrium.

\section{TABle 2}

Proportions of male steriles in generation 500 when male sterility is digenically inherited with gene interaction (13 $\mathrm{H}: 3 \mathrm{MS}$ in $\mathrm{F}_{2}$ )

Initial

proportions

of Initial gene

male frequencies

steriles $p m s_{1}, p m s_{2}$

$0.920126 \quad 0.96,0.96 \quad 0.00$

$w=1$

$w=2$

$w=2 \cdot 01$

$w=3$

$w=10$

$w=100$

0.4851

$0.9,0.7 \quad 0.000016$ *

$0.008515 *$

0.010763 *

$0 \cdot 25 * * *$

$0.444445 \dagger$

$0.444446 \dagger$

$0.010781^{*} \quad 0.25^{* * *}$

$0 \cdot 444446 * * *$

0.494950***

$0 \cdot 1875$

$\begin{array}{lll}0.5, & 0.5 & 0.000027 *\end{array}$

$0.009802 *$

$0.011996^{*}$

$0 \cdot 25^{* * *}$

$0.444446 * * *$

$0.494951 \dagger$

0.0099

$\begin{array}{lll}0.9, & 0 \cdot 1 & 0 \cdot 000015^{*} \\ 0.04,0.04 & 0.000036^{*}\end{array}$

$0.005606^{*}$

$0.007374 *$

$0.35 * * *$

$0.444445^{* * * *}$

$0 \cdot 494950$ ***

0.000125*

$0.000128 * * \quad 0 \cdot 25 \dagger \dagger$

$0.494951 \uparrow$

* Decreasing. **Increasing. *** At equilibrium.

$\dagger$ Fluctuating slightly, apparently very near equilibrium.

t† 0.249999859 increasing very slowly.

TABle 3

Proportions of male steriles in generation 500 when male sterility is digenically inherited with gene interaction ( $9 \mathrm{H}: 7 \mathrm{MS}$ in $\mathrm{F}_{2}$ )

Initial

proportions

of Initial gene

male frequencies

steriles $\quad p m s_{1}, p m s_{2}$

$p m s_{1}, p m s_{2}$
$0.9, \quad 0.9$

$w=1$

$w=2$

$w=2 \cdot 01$

$w=3$

$w=10$

$w=100$

0.9639

$\begin{array}{lll}0.3, & 0.5 & 0.000032 * \\ 0.1, & 0.1 & 0.000030 *\end{array}$

$0.010589 *$

0.012760 *

$0 \cdot 25^{* * *}$

$0 \cdot 444445 * * *$

$0 \cdot 494950 * * *$

0.3175

$0 \cdot 1, \quad 0 \cdot 1 \quad 0 \cdot 000030$ *

$0.008283^{*}$

$0.012598 *$

$0 \cdot 25 * * *$

$0.444445 * * *$

$0 \cdot 494950 * * *$

0.0002

$0.01,0.01 \quad 0.000016^{*}$

$0 \cdot 000199 * 0 \cdot 000209 * *$

$0 \cdot 25 * * * \quad 0 \cdot 444445 * * *$

$0 \cdot 494950 * * *$

0.494950***

* Decreasing. ** Increasing. ***At equilibrium.

as to suggest the ultimate loss of the male steriles from the population. With a relative fertility of two, declines in the proportions of male steriles were much slower, while a $w$ value of 2.01 produced an increase in the lowest starting 
value only, suggesting the presence of a low equilibrium value for the proportion of male steriles. Relative fertility values of 3,10 and 100 produced equilibrium values of $0.25,0.444444$ and 0.494949 respectively. Similar results were obtained where the male sterility was dominant, except that the male steriles were compeltely lost from the population when their fertility as females was equal to that of the hermaphrodites.

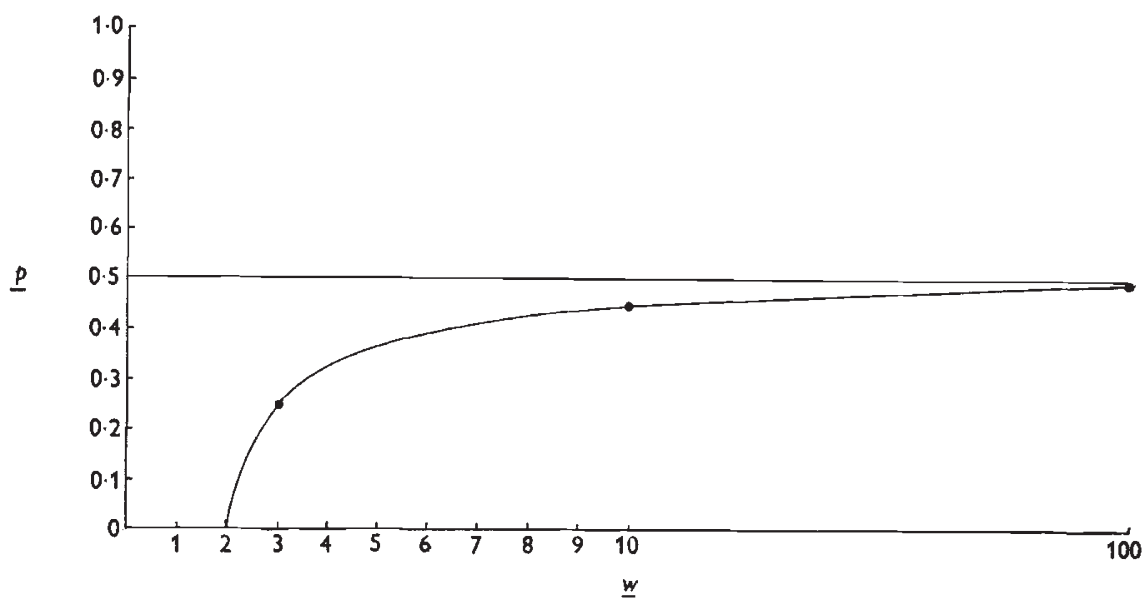

FIG. 1.-Relation between the relative female fertility of male steriles $(w)$ and their equilibrium proportion $(p)$.

With digenic inheritance of male sterility three types of gene interaction were studied, producing respectively in $\mathrm{F}_{2}$ 15H: $1 \mathrm{MS}$ (table 1), 13H: $3 \mathrm{MS}$ (table 2) and 9H: 7MS (table 3). From these tables it is clear that the results are very similar to those under monogenic inheritance. There were declines in all starting values for the two lowest relative fertility values $(w=1$ and $w=2$ ) while again only the lowest starting values showed increases for $w=2 \cdot 01$. The higher values for relative fertility of the male steriles produced essentially identical equilibria to those found under monogenic inheritance. The relation between equilibrium proportions of male steriles and their relative fertilities under the five systems of inheritance considered here is given graphically in fig. 1 .

\section{Discussion}

We have followed Lewis (loc. cit.) in studying the effects of varying the relative seed fertility of the male steriles on their equilibrium proportions. However, it is clear that other means of maintaining male steriles in populations may exist. For example, heterozygote advantage could assist in such a function (Jain, 1961). However, a statement of the relative seed fertility required to maintain the male steriles in a population is useful for the purpose of comparing our results with those of Lewis.

Our data indicate that the increase in relative seed fertility required to maintain the male steriles in the population is probably independent of the number of genes controlling the male sterility or of their mode of interaction. Instead the requirement for doubled fertility of the male steriles seems to be a consequence of the fact that the male steriles can contribute genes to the 
next generation in only one of the two ways available to the hermaphrodites. This fact is in turn a consequence of the inheritance of the male sterility through the nucleus. When we consider male sterility transmitted solely through the maternal cytoplasm, the requirement for greater seed fertility of the male steriles virtually disappears (Lewis, 1941). In respect of characters not transmitted through the pollen the male steriles are the equals of the hermaphrodites.

Our conclusion that the selective pressures required to maintain male steriles under digenic inheritance are no greater than those required under monogenic inheritance removes one of the reasons for surprise that digenic systems should occur in nature (cf. Lewis and Crowe, loc. cit.). Nevertheless, an important evolutionary problem remains. Since male-sterile mutants are frequent in the flowering plants, and since such mutants are usually inherited as monogenic recessives, one would expect male sterility in gynodioecious plants to have developed from such mutations, and to show monogenic inheritance also. Why then are digenic systems found? Lewis and Crowe (loc. cit.) sought to derive the digenic system in Origanum from a selfincompatibility gene together with a dominant mutation for male sterility. A possible alternative explanation, which could be applied to the Plantago system also, might be found in the cumulative heterosis model of Lewontin (1964). Under this model, with two heterotic loci, the degree of heterosis is not additive. Instead, the amount of heterosis is greater at the first locus when the second locus is also heterozygous.

Another point of interest lies in the equilibria attained by the male steriles under various fertility values. Lewis' expression for the relation between the proportion $p$ of male steriles at equilibrium and their relative fertility $f$ is

$$
p=\frac{1}{2}-\frac{\mathrm{I}}{f(\mathrm{I}-p)^{x-1}}
$$

where $x$ is an unknown. The value of $x$ does not affect the requirement for doubled fertility of the male steriles. However, it does affect the equilibrium proportion of male steriles $p$ for a given relative fertility $f$. Thus when $x=0$ and $f=3, p=0.25$, and when $x=1$ and $f$ remains at $3, p=0.167$. Our results are consistent with a value of $x=0$, but Lewis (loc. cit.) held that the true value of $x$ was between 0.5 and 2 .

Our results gave the same equilibrium values under digenic as under monogenic inheritance. If we are justified in assuming that these equilibria hold for cases of trigenic and polygenic inheritance also, then we may conclude that the limiting proportion of 0.5 male steriles applies to these cases, and perhaps to all types of nuclear inheritance. Lewis has already shown that equilibria of more than 0.5 male steriles may exist under cytoplasmic inheritance. Consequently, if we obtain field counts of more than 50 per cent. male steriles, and if we have evidence that the populations are at equilibrium, then we may take these counts as indirect evidence for a cytoplasmic type of inheritance of the male sterility. However, we should be cautious because complications not considered here or by Lewis may allow equilibrium proportions greater than 0.5 even under nuclear inheritance. Nevertheless, there is some evidence to suggest that an association between high proportions of male steriles and cytoplasmic inheritance of the male sterility may exist. There are a number of counts of around 50 per cent. 
male steriles in the literature, e.g. 53 per cent. in one population of Cortaderia richardii (Connor, loc. cit.). However, there appear to be only a few counts of considerably over 50 per cent. male steriles. One of these is a 65 per cent. male steriles recorded by Chandler (1954) in plants derived from strains of Plantago ovata cultivated in India. It is interesting that inheritance of male sterility in Plantago ovata seems to be cytoplasmic (Paliwal and Hyde, 1959). Another case of very high proportions of male steriles also occurs in a plantain, Plantago lanceolata. Here proportions of up to 70 per cent. male steriles have been recorded by Baker (1963). In this species there appears to be some factor controlling the inheritance of male sterility in addition to the two genes posturated (Ross, loc. cit.).

There is a great lack of data on proportions of male steriles in natural populations of gynodioecious plants. In the present context counts of the species where inheritance of male sterility seems to be cytoplasmic would be particularly welcome (Cirsium oleraceum, C. acaule, Satureia hortensis and Plantago ovata).

It is convenient at this point to discuss briefly a statement made by Lewis and Crowe (1956) that a hermaphrodite contributes three times as many genes to the next generation as does a male sterile, since the hermaphrodite supplies its own pollen and egg nuclei and also the pollen nucleus that fertilises the male sterile. It can be seen that this statement is valid in one case only, namely, when the number of male steriles in a population equals the number of hermaphrodites. The relative contribution of genes to the next generation depends partly on the proportion of male steriles. Consider a population of 100 plants of an annual species such that one seed from each plant survives to form the next generation. If there are 99 male steriles and 1 hermaphrodite, then 99 sets of genes are contributed to the next generation via the eggs of the male steriles. Ninety-nine sets of genes are also contributed by the hermaphrodite that fertilises these eggs, and two further sets are contributed when the hermaphrodite is selfed, to give a total contribution from the hermaphrodite of 101 sets. Thus the contributions of the male steriles and the hermaphrodite are nearly equal; indeed they are equal if we vary the conditions by making the hermaphrodite self-incompatible. The one hermaphrodite under these conditions contributes about 100 times as many sets of genes as any one male sterile. With a population of 50 male steriles and 50 hermaphrodites the contributions of the male steriles and the hermaphrodites are 50 and 150 sets of genes respectively. Here one hermaphrodite contributes three times as many genes as one male sterile. When there is 1 male sterile and 99 hermpahrodites, the relative contributions are $1: 199$, and any one hermaphrodite contributes about twice as many genes as the one male sterile. This example incidentally demonstrates the presence of a type of frequency-dependent selection, since the male steriles have a higher selective value when they are rare.

\section{Summary}

1. Gynodioecious plants consist of male steriles (i.e. females) and hermaphrodites, and gynodioecy is an outbreeding mechanism because of the enforced outcrossing of male steriles to hermaphrodites.

2. An earlier conclusion that under monogenic inheritance of male sterility male steriles are lost from the population unless they are at least 
twice as seed fertile as hermaphrodites, is confirmed and extended to digenically-inherited male sterility with three modes of gene interaction.

3. Proportions of male steriles in equilibrium populations are the same for given relative fertility values under monogenic and under digenic inheritance.

\section{REFERENGES}

BAKER, H. G. 1963. Evolutionary mechanisms in pollination biology. Science, 139, 877-883. CHANDLER, c. 1954. Improvement of Plantago for mucilage production and growth in the United States. Contrib. Boyce Thompson Inst., 17, 495-505.

ConNor, H. E. 1965. Breeding systems in New Zealand grasses. VI. Control of gynodioecism in Cortaderia richardii (Endl.) Zotov. New Zealand 7. Bot., 3, 233-242.

JAIN, s. K. 1961. On the possible adaptive significance of male sterility in predominantly inbreeding populations. Genetics, 46, 1237-1240.

LEWIS, D. 1941. Male sterility in natural populations of hermaphrodite plants. New Phytol., $40,56-63$.

LEWTS, D., AND CROWE, L. K. 1956. The genetics and evolution of gynodioecy. Evolution, 10, 115-125.

LEWONTIN, R. C. 1964. The interaction of selection and linkage. I. General considerations; heterotic models. Genetics, 49, 49-67.

MATHER, K. 1940. Outbreeding and separation of the sexes. Nature, 145, 484-486.

PALIWAL, R. L., AND HYDE, B. B. 1959. Association of a single B-chromosome with male sterility in Plantago coronopus. Amer. 7. Bot., 46, 460-466.

Ross, M. D. 1969. Digenic inheritance of male sterility in Plantago lanceolata. Can. F. Genet. Cytol., 11, 739-744.

sHAw, R. F. 1957. The theoretical genetics of the sex ratio. Genetics, 43, 149-163. 